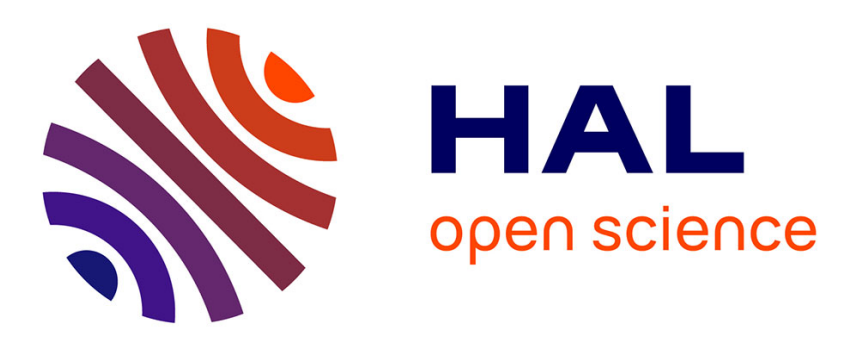

\title{
Améliorer la mobilité des enseignants sans pénaliser les académies les moins attractives?
}

Julien Combe, Olivier Tercieux, Camille Terrier

\section{To cite this version:}

Julien Combe, Olivier Tercieux, Camille Terrier. Améliorer la mobilité des enseignants sans pénaliser les académies les moins attractives?. 2016. halshs-02522857

\section{HAL Id: halshs-02522857 \\ https://shs.hal.science/halshs-02522857}

Submitted on 27 Mar 2020

HAL is a multi-disciplinary open access archive for the deposit and dissemination of scientific research documents, whether they are published or not. The documents may come from teaching and research institutions in France or abroad, or from public or private research centers.
L'archive ouverte pluridisciplinaire HAL, est destinée au dépôt et à la diffusion de documents scientifiques de niveau recherche, publiés ou non, émanant des établissements d'enseignement et de recherche français ou étrangers, des laboratoires publics ou privés. 


\section{AMÉLIORER LA MOBILITÉ DES ENSEIGNANTS SANS PÉNALISER LES ACADÉMIES LES MOINS ATTRACTIVES ? LE RÔLE CLÉ D’UN NOUVEL ALGORITHME D’AFFECTATION}

\section{Les notes de I'IPP}

$n^{\circ} 24$

Avril 2016

\section{Julien Combe Olivier Tercieux Camille Terrier}

\section{www.ipp.eu}

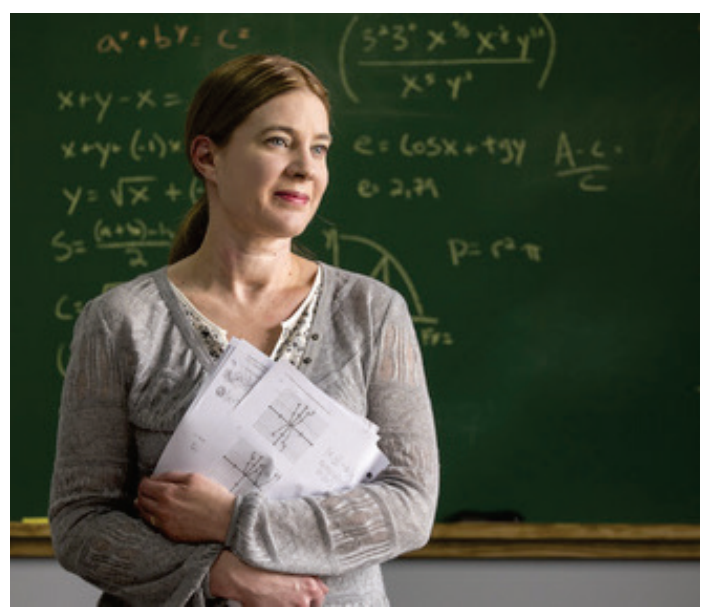

\section{Résumé}

L'affectation des enseignants aux établissements des premier et second degrés recouvre une multitude d'enjeux importants : attractivité de la profession, inégalités géographiques, réussite des élèves, etc. L'arbitrage entre ces différentes dimensions s'avère délicat: assurer une mobilité forte des enseignants peut se traduire par une augmentation des inégalités entre académies en termes d'expérience des enseignants affectés, et ainsi pénaliser la réussite des élèves dans les académies les moins attractives. Dans ce contexte, la procédure informatique utilisée en France pour affecter les enseignants représente un levier d'action essentiel pour réaliser l'arbitrage entre la satisfaction des vœux de mutation et l'égalité des territoires. Partant du constat que la procédure actuelle tend à limiter la mobilité des enseignants, nous proposons dans cette note une procédure d'affectation alternative qui permettrait d'augmenter de plus de $30 \%$ le mouvement des enseignants titulaires tout en prenant en compte les spécificités des académies les moins attractives. Pour ces académies, nous fournissons un outil de pilotage qui permet de faire des simulations et de tester différentes stratégies de gestion des ressources humaines - augmentation, maintien ou diminution du mouvement dans ces académies. Même si le choix final dépend d'une concertation entre les différents acteurs sur les objectifs à atteindre, notre travail souligne l'impact positif important que pourrait avoir une modification du système actuel d'affectation des enseignants.

- La procédure informatique utilisée en France pour affecter les enseignants souffre d'un problème qui limite fortement le mouvement chaque année : en 2013, le mouvement inter-académique du second degré aurait pu être $40 \%$ plus élevé au niveau national, et jusqu'à près de $150 \%$ pour certaines académies si un algorithme plus efficace avait été utilisé.

- Une hausse du mouvement - sans précautions additionnelles - peut accroître la surreprésentation des jeunes enseignants dans les académies les plus défavorisées et, plus généralement, augmenter les inégalités entre les différentes académies en termes d'expérience des enseignants affectés.

- Nous fournissons un outil de pilotage qui permet de faire des simulations et de tester différentes stratégies de gestion des ressources humaines - augmentation, maintien ou diminution du mouvement - propres à chaque académie.

- À titre d'exemple, nous montrons qu'une procédure alternative d'affectation permettrait d'augmenter de plus de $30 \%$ le nombre d'enseignants obtenant une nouvelle affectation tout en maintenant constant le mouvement dans les académies défavorisées. Dans ce scénario, le mouvement dans certaines académies serait multiplié par deux.
L'Institut des politiques publiques (IPP) est développé dans le cadre d'un partenariat scientifique entre PSE et le CREST. L'IPP vise à promouvoir l'analyse et l'évaluation quantitatives des politiques publiques en s'appuyant sur les méthodes les plus récentes de la recherche en économie.
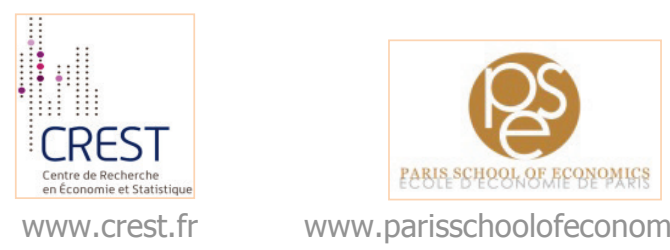

www.parisschoolofeconomics.eu 


\section{L'affectation des enseignants : une procédure aux enjeux importants}

La plupart des systèmes éducatifs font face à deux problèmes : le manque d'attractivité de la profession enseignante, d'une part, et de fortes inégalités de réussite entre élèves liées en grande partie à leur origine sociale, d'autre part. Un exemple français illustre le premier point : $24 \%$ des postes au Capes externe n'ont pas été pourvus en 2014. Concernant les inégalités de réussite, les résultats de l'enquête PISA 2012 ont fait apparaître que la France est le pays de I'OCDE où le milieu social conditionne le plus la réussite scolaire des élèves ${ }^{1}$. Bien que ces inégalités renvoient à une multiplicité de facteurs explicatifs, elles tendent à être accentuées par le fait que les académies les plus défavorisées socialement sont aussi celles qui accueillent les enseignants les moins expérimentés. À Créteil et Versailles, par exemple, on observe à la fois (i) une très forte proportion de collégiens scolarisés en éducation prioritaire $^{2}$ et (ii) la plus faible proportion d'enseignants de 50 ans et plus relativement aux moins de 30 ans. Les performances académiques des élèves étant significativement moins bonnes lorsque leurs enseignants viennent d'être recrutés (néo-titulaires) ou n'ont qu'une ou deux années d'expérience, la surreprésentation des jeunes enseignants dans les académies les plus défavorisées contribue certainement significativement aux inégalités de réussite ${ }^{3}$.

La question de l'affectation des enseignants se situe au croisement de ces deux problématiques. Du point de vue de I'attractivité du métier d'enseignant, les perspectives de mobilité géographiques sont un facteur clé. À cet égard, un objectif naturel pourrait être de satisfaire le plus possible les demandes de mutations des enseignants. Malheureusement, les demandes de mobilité sont, dans le système actuel, majoritairement refusées. En 2014, seuls 43,9\% des enseignants titulaires ayant fait une demande de mobilité entre académies ont obtenu satisfaction ${ }^{4}$. Notre étude montre qu'il est possible d'améliorer le système actuel dans cette dimension : nous expliquons comment une simple modification de la procédure automatisée - aussi appelée algorithme - peut significativement augmenter le taux de satisfaction des demandes de mobilité des enseignants.

La question de l'affectation des enseignants est également au cœur de la problématique des inégalités de réussite, comme le souligne le récent rapport de l'Inspection générale de l'administration de l'éducation nationale et de la recherche (IGAENR) ${ }^{5}$. Dans ce contexte, le processus centralisé utilisé par le ministère et les rectorats pour affecter les enseignants dans les académies, puis dans les établissements, constitue un levier d'action puissant pour homogénéiser la répartition des enseignants entre académies, garantir une plus grande stabilité des équipes et prendre en compte les besoins pédagogiques des établissements. Or, comme le souligne le rapport de I'IGAENR, « la question de l'égalité RH des territoires est peu observée et peu analysée ». Notre étude souligne que le processus centralisé d'affectation est un outil encore sousexploité pour répondre à ces problématiques.

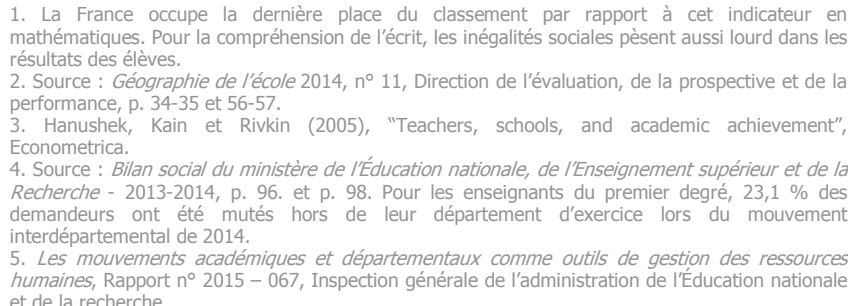

Il faut cependant noter que les deux objectifs de satisfaction des demandes de mutation et de répartition plus équilibrée des enseignants sur le territoire en fonction de leur niveau d'expérience peuvent apparaître comme difficilement compatibles. Une augmentation du mouvement peut se faire au prix d'une augmentation des inégalités entre académies du point de vue de l'expérience moyenne des enseignants affectés. En effet, dans la mesure où la plupart des demandes de mobilité émanent d'académies au profil social plutôt défavorisé (notamment les académies d'Amiens, de Créteil et de Versailles), accroître la mobilité des enseignants peut avoir pour effet d'augmenter les flux sortants de ces académies, qu'il faut compenser par des flux entrants plus importants, constitués majoritairement de jeunes enseignants ${ }^{6}$. Partant de ce constat de l'existence d'un arbitrage entre mobilité des enseignants et égalité des territoires, la contribution de notre étude est de proposer un algorithme qui permet d'améliorer le mouvement tout en évitant de pénaliser les académies les plus défavorisées.

\section{L'affectation des enseignants dans les académies : un processus centralisé}

En 2013, on comptait 487500 enseignants dans le second degré en France 7 . Pour les 382600 enseignants du secteur public, une procédure centralisée est utilisée pour gérer les affectations dans les établissements. Depuis 1999, cette procédure fait intervenir deux phases distinctes : (1) le mouvement inter-académique, d'une part, qui affecte les enseignants dans les 31 académies ; (2) le mouvement intra-académique, d'autre part, qui affecte les enseignants aux différents établissements d'une même académie. En 2013, environ 25000 enseignants ont participé au mouvement interacadémique et 65000 enseignants ont participé au mouvement intra-académique.

Notre étude porte exclusivement sur le mouvement interacadémique $^{8}$. Pendant cette phase, les enseignants souhaitant changer d'académie et les enseignants nouvellement recrutés soumettent à l'administration centrale une liste ordonnée de 1 à 31 académies dans lesquelles ils aimeraient être affectés. Au sein de chaque académie, les candidats sont ordonnés au moyen d'un barème défini par le ministère. Ce barème tient compte de trois priorités légales (le rapprochement de conjoints, la situation de handicap et l'enseignement en éducation prioritaire), ainsi que des caractéristiques personnelles des enseignants telles que I'ancienneté dans l'enseignement/le poste, les années passées à distance du conjoint, etc.

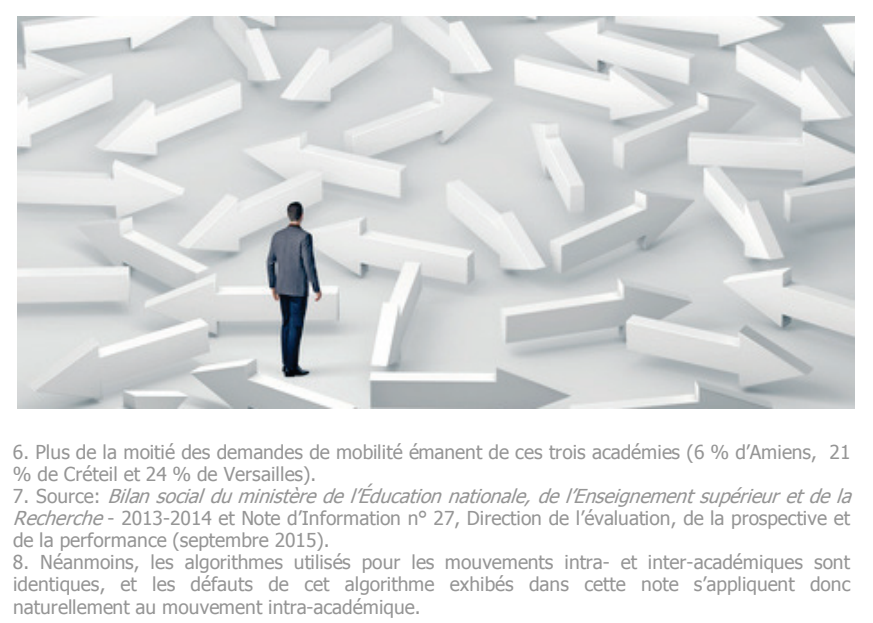




\section{L'algorithme utilisé actuellement tend à limiter la mobilité des enseignants}

À partir des préférences des enseignants (les vœux formulés) et du barème de ces derniers dans chacune des académies, le ministère utilise une procédure automatisée - un algorithme qui affecte les enseignants dans les différentes académies. Cet algorithme correspond à une version légèrement modifiée de l'un des algorithmes les plus fréquemment utilisés pour affecter les élèves dans les établissements ${ }^{9}$, appelé « algorithme d'acceptation différée ${ }^{10} \gg($ voir encadré 1$)$.

\section{Encadré 1 : L'algorithme dit « d'acceptation différée »}

A partir des vœux formulés par les enseignants, et de leurs barèmes dans les différentes académies, I'algorithme d'acceptation différée fait intervenir une série d'étapes qui sont mises en œuvre de manière automatisée en s'appuyant uniquement sur les vœux de mobilité des enseignants et sur l'application mécanique des règles de priorité :

- Étape 1 : tous les enseignants candidatent dans l'académie qu'ils classent en premier vœu. Les académies acceptent temporairement les candidats par ordre décroissant de barème dans la limite des places disponibles, et rejettent les autres candidats. Un point important est que l'acceptation est temporaire.

- Étape 2 : tous les candidats rejetés à l'issue de la première étape candidatent dans l'académie la mieux classée parmi celles auxquelles ils n'ont pas encore candidaté. Les académies considèrent conjointement (1) les nouvelles candidatures et (2) les candidats temporairement retenus à l'étape 1 . Parmi cet ensemble d'enseignants, les académies acceptent temporairement les candidats par ordre décroissant de barème dans la limite de leurs places disponibles, et rejettent les autres. Un candidat peut donc avoir été temporairement accepté à l'étape 1 , mais rejeté à l'étape 2 du fait de la candidature de nouveaux enseignants disposant d'un barème plus élevé.

- Le processus se termine après un nombre fini d'étapes, lorsqu'aucun enseignant n'est plus rejeté par une académie. L'acceptation des académies est alors définitive et détermine l'affectation finale des enseignants.

Une contrainte importante doit être prise en compte par la procédure d'affectation des enseignants : la plupart des participants au mouvement étant des enseignants titulaires initialement affectés dans une académie (70 \% pour le mouvement inter-académique), ils doivent pouvoir rester dans leur académie d'origine s'ils n'obtiennent aucune de celles qu'ils ont classées dans leur liste de vœux. Par exemple, un enseignant initialement affecté dans l'académie de Grenoble et qui ne formulerait que deux vœux d'affectation (Bordeaux et Toulouse) doit pouvoir rester à Grenoble s'il n'obtient aucun de ces deux vœux. Cette dernière condition correspond au droit à rester dans son académie d'origine. Pour garantir ce droit, l'algorithme utilisé en France pour gérer le mouvement inter-académique est une version modifiée de « l'algorithme d'acceptation différée ». La modification consiste à ajouter, pour les enseignants qui participent à la procédure tout en étant déjà en poste dans une académie, un vœu « fictif » pour leur académie d'origine, qui est placé en dernière position dans leur liste de vœux, et à leur attribuer un barème maximal pour ce vœu. L'affectation est réalisée en appliquant l'algorithme précédent aux listes de vœux et aux priorités ainsi modifiées ${ }^{11}$.
Comme nous l'avons déjà souligné, la proportion d'enseignants titulaires dont les demandes de mutation sont satisfaites est faible, tant dans le premier que dans le second degré. À l'aide d'un exemple simple (voir encadré 2), nous mettons en évidence une des raisons essentielle de ce défaut de mobilité. En particulier, nous illustrons qu'avec l'algorithme actuel, un faible mouvement peut être facilement observé lorsqu'un enseignant doté d'un barème élevé demande à sortir d'une académie relativement peu attractive pour les autres enseignants $^{12}$.

\section{Encadré 2 : Pourquoi l'algorithme actuel nuit-il à la mobilité ?}

Vœux des enseignants. Les défauts de l'algorithme actue peuvent être illustrés à partir d'un exemple simple. Considérons une situation où seuls trois enseignants participent au mouvement interacadémique : Arthur, Bertrand et Victoria, qui sont initialement affectés à Amiens, Bordeaux et Versailles respectivement. Victoria et Bertrand ne formulent qu'une seule demande de mutation pour respectivement l'académie de Bordeaux et de Versailles. De son côté, Arthur formule deux demandes de mutations, l'une à Versailles et l'autre à Bordeaux. Les demandes de mutations sont représentées de manière schématique dans la carte cicontre.

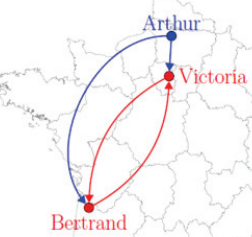

Barème des académies. Pour ces trois enseignants, nous supposons que les priorités sont déterminées uniquement par l'expérience et que, pour toutes les académies, Arthur a priorité sur Bertrand, qui a lui-même priorité sur Victoria.

L'absence de mouvement. À partir des vœux des enseignants et de leurs barèmes (modifiés de sorte à assurer le droit à rester dans son académie d'origine), les différentes étapes décrites dans l'encadré 1 permettent d'obtenir l'affectation des enseignants dans les académies. Comme l'algorithme actuel donne droit à rester dans son académie d'origine et qu'aucun enseignant n'est prêt à remplacer Arthur, Arthur devra rester à Amiens. Dans l'algorithme actuel, il postulera donc à Versailles et Bordeaux mais en sera rejeté. En revanche, le fait même qu'il postule dans ces académies empêche Victoria et Bertrand de voir leurs demandes de mutation satisfaites. En effet, Victoria se verra rejetée de I'académie de Bordeaux car Arthur - qui dispose d'un barème plus élevé - y postule également. De même, Bertrand ne pourra obtenir sa mutation car Arthur postule à Versailles.

Le « paradoxe » de la situation. Les demandes de mutations de Victoria et Bertrand ne peuvent pas être satisfaites à cause des deux candidatures d'Arthur alors même que ces dernières seront toutes rejetées ! Cette situation se traduit donc par une absence complète de mouvement alors que, pour Victoria et Bertrand, « échanger » leurs affectations augmenterait leur satisfaction tout en homogénéisant la répartition des enseignants expérimentés entre académies - en transférant un enseignant relativement plus expérimenté (Bertrand) de Bordeaux à Versailles.

Conclusion. Il est donc possible, à travers ces échanges, d'augmenter le mouvement par rapport à la situation actuelle tout en diminuant les inégalités entre académies. L'objectif de notre étude est de mesurer, à partir des données réelles du mouvement inter-académique du second degré, le surcroît de mobilité que permettraient de tels échanges. 


\section{Il est possible d'améliorer le mouvement}

Bien que spécifique, l'exemple décrit dans l'encadré 2 illustre une faiblesse importante de l'algorithme actuel du point de vue de la mobilité des enseignant. Pour mesurer empiriquement l'ampleur réelle de ce phénomène, nous avons utilisé les données sur les vœux de mobilité et le barème de priorité des enseignants pour reproduire les affectations obtenues avec l'algorithme actuel. La faiblesse de l'algorithme se retrouve de façon marquée lorsque nous analysons le mouvement interacadémique du second degré. Les résultats de nos simulations (voir tableau 1) montrent que le mouvement pour les enseignants titulaires pourrait être augmenté de près de $40 \%$ si l'on modifiait l'algorithme actuel en introduisant le système dit $d^{\prime} \ll$ échanges mutuellement améliorants » (EMA) brièvement décrit à la fin de l'encadré 2 (et en ignorant dans un premier temps les difficultés qu'une augmentation du mouvement peut causer du point de vue des inégalités entre académies). Cette augmentation de la mobilité concernerait la quasi-totalité des matières enseignées : elle serait de $36 \%$ en mathématiques, de $34 \%$ en lettres modernes et de $51 \%$ en anglais. Elle serait également observée dans toutes les académies (voir carte 1 ).

Carte 1 : Surcroît de mobilité par rapport à l'algorithme actuel (en \%)

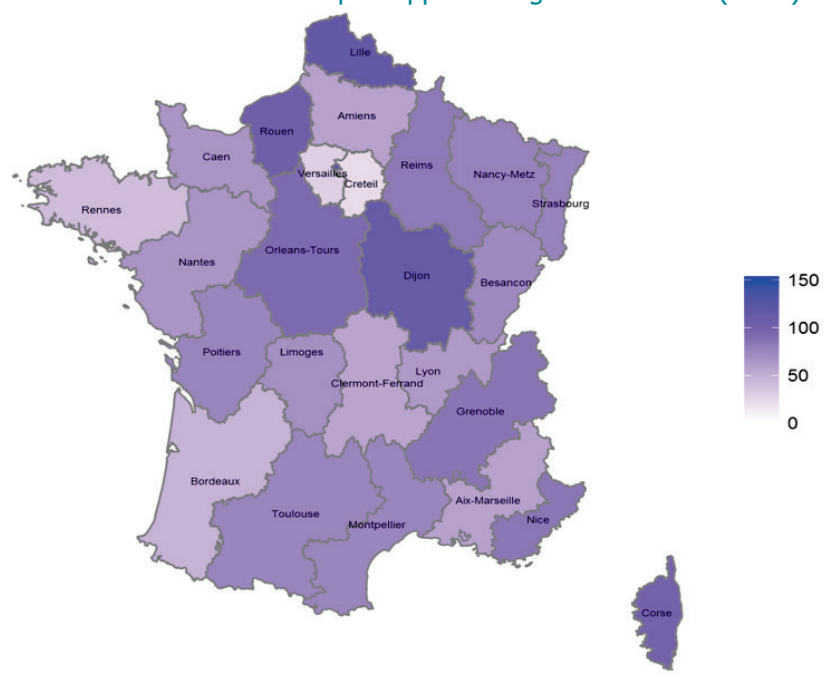

\section{Option 1}

\section{Prise en compte des académies prioritaires}

Comme nous l'avons déjà mentionné, augmenter la mobilité des enseignants peut se traduire par des sorties importantes pour les académies les moins attractives, qu'il faut compenser par des entrées de même ampleur et majoritairement constituées de jeunes enseignants. Cette situation peut conduire à une augmentation de la proportion relative d'enseignants peu expérimentés dans les académies les plus socialement défavorisées.

Or, toute augmentation de la mobilité des enseignants qui se fait au détriment des académies les moins attractives peut apparaître comme problématique. Nos simulations montrent que les sorties des académies de Créteil et Versailles passeraient respectivement de 813 et 779 avec l'algorithme actuel à 995 et 1002 avec le premier algorithme alternatif que nous suggérons. L'introduction de ce nouvel algorithme augmenterait par ailleurs la proportion de néo-titulaires parmi les entrants dans ces académies de $9 \%$ à Créteil et $11 \%$ à Versailles. Une contribution de notre étude est de proposer un algorithme d'affectation qui permette d'éviter de pénaliser de la sorte les académies les moins attractives.
Un préalable à la mise en œuvre de cet algorithme est d'identifier les « académies prioritaires », c'est-à-dire les académies pour lesquelles des sorties importantes chaque année peuvent s'avérer problématiques ${ }^{13}$. Une fois défini ce groupe d'académies prioritaires, notre algorithme a pour but d'identifier les possibilités d'échanges entre enseignants qui permettent d'augmenter le mouvement sans pénaliser les académies prioritaires (voir encadré 3 ). Une première approche consiste à exiger que, dans les académies prioritaires, tout enseignant sortant soit forcément remplacé par un enseignant entrant disposant d'un barème de priorité plus élevé.

Dans l'exemple décrit dans l'encadré 2, en supposant qu'Amiens et Versailles sont des académies prioritaires et en partant de l'affectation initiale, il est clair qu'il existe un échange améliorant entre Victoria et Bertrand ${ }^{14}$. Notre algorithme affectera donc Victoria à Bordeaux et Bertrand à Versailles. Arthur restera à Amiens, comme dans le cas de l'algorithme actuel. Au-delà de cet exemple, un certain nombre de nos résultats théoriques suggèrent que les échanges mutuellement améliorants produisent de meilleurs résultats que l'algorithme utilisé actuellement du point de vue du mouvement des enseignants, tout en tenant compte de la situation de certaines académies identifiées comme prioritaires.

Encadré 3 : Présentation de l'algorithme $d^{\prime}$ 《 échanges mutuellement améliorants » avec académies prioritaires ${ }^{15}$

A chaque étape de l'algorithme, nous définissons pour chaque enseignant l'ensemble des académies pour lesquels il est dit « éligible ». Pour déterminer si un enseignant est éligible à une académie, deux cas doivent être distingués :

1. L'académie n'est pas prioritaire : l'enseignant y est automatiquement éligible si certains enseignants en poste dans cette académie ne sont pas encore affectés.

2. L'académie est prioritaire : pour que l'enseignant y soit éligible, il doit disposer d'un barème plus élevé que certains des enseignants en poste dans cette académie, faisant une demande de mutation et n'ayant pas été encore affectés par l'algorithme.

A chaque étape de l'algorithme, un échange mutuellement améliorant (EMA) existe si un ensemble d'enseignants peuvent tous échanger leurs académies et : (1) l'académie obtenue par chaque enseignant dans cet

échange est son académie préférée parmi les académies auxquelles il est éligible, et (2) si dans cet échange, un enseignant prend le poste d'un autre enseignant dans une académie prioritaire, alors il doit avoir un barème supérieur à ce dernier dans cette académie ${ }^{16}$.

À partir des vœux formulés par les enseignants, et de leur barème dans les différentes académies, l'algorithme consiste à appliquer de manière itérative les étapes suivantes

- Étape 1: À partir de l'affectation initiale, nous définissons pour chaque enseignant les académies pour lesquelles il est éligible. Nous identifions un échange mutuellement améliorant. L'échange identifié est réalisé et un enseignant faisant partie de l'échange est définitivement affecté à l'académie qu'il obtient. Un enseignant dont les académies auxquelles il est éligible n'ont plus de place est considéré comme définitivement affecté à son académie d'origine.

- Étape 2: Nous considérons uniquement les enseignants non affectés à l'étape 1 , et nous définissons pour chaque enseignant les académies pour lesquelles il est éligible. Nous identifions un échange mutuellement améliorant. L'échange identifié est réalisé et un enseignant faisant partie de l'échange est définitivement affecté à l'académie qu'il obtient. Un enseignant dont les académies auxquelles il est éligible n'ont plus de place est considéré comme définitivement affecté à son académie d'origine.

- Le processus se poursuit jusqu'à ce qu'il n'existe plus d'échanges améliorants.

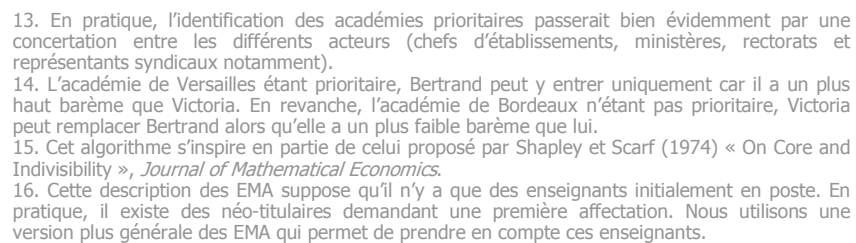


Pour tester la validité de ces résultats théoriques ${ }^{17}$, nous sommes partis des données sur les vœux de mobilité et le barème de priorité des enseignants pour simuler les affectations obtenues au moyen des échanges mutuellement améliorants lorsque certaines académies sont prioritaires, et les avons comparées avec les performances du système actuel, et celles des simulations précédentes dans lesquelles aucune des académies n'était considérée comme prioritaire. Dans nos simulations, nous avons choisi d'attribuer le statut d'académie prioritaire à Amiens, Créteil et Versailles. Nos résultats indiquent que le mouvement global continue à augmenter significativement par rapport à la situation actuelle $(\mathbf{+ 1 5 \%} \%$ ). La nouvelle condition, qui impose que tout enseignant sortant soit remplacé par un enseignant disposant d'un barème plus élevé, implique naturellement que le rapport entre le nombre de titulaires sortants et entrants se rapproche de 1 dans les académies prioritaires et que l'âge moyen et la proportion d'enseignants titulaires augmente dans ces académies. Néanmoins, et expliquant partiellement ce phénomène, le nombre d'enseignants sortants des académies de Créteil et Versailles diminue (respectivement de 504 et 319).

\section{Option 2}

\section{Un mouvement finement ciblé pour les académies prioritaires}

La diminution des flux sortants des académies les moins attractives pouvant être perçue comme problématique, une solution consiste à ne pas donner priorité à l'intégralité d'une académie, mais seulement à certains d'enseignants (qui peuvent être sélectionnés en fonction de leur barème). En d'autres termes, cette solution consiste à identifier, au sein des académies prioritaires, des enseignants « cibles » qui, pour sortir de l'académie, n'auront pas besoin d'être remplacés par un enseignant ayant un barème plus élevé. Relâcher la contrainte que tout enseignant quittant une académie prioritaire doive être remplacé par un enseignant à plus haut barème permet un calibrage fin des flux de sorties des académies prioritaires.

Pour chacune des trois académies prioritaires, nous avons calculé le pourcentage d'enseignants pour lesquels la contrainte doit être relâchée si l'on souhaite conserver les mêmes mouvements de sorties de ces académies que dans le système d'affectation actuel.
En relâchant la contrainte pour $60 \%$ des enseignants de Créteil, $45 \%$ des enseignants de Versailles et $40 \%$ de ceux d'Amiens, les mouvements de sorties de ces trois académies restent identiques ou s'améliorent légèrement. Nous simulons les affectations obtenues pour cette nouvelle version de notre algorithme. Le nombre d'enseignants titulaires obtenant une nouvelle académie continue à augmenter significativement par rapport à la situation actuelle (de $32 \%$ en moyenne, voir tableau 2), et ce dans toutes les académies : le pourcentage d'enseignants titulaires obtenant leur mutation passe ainsi de $34 \%$ à $75 \%$ dans l'académie de Paris, de $40 \%$ à $85 \%$ à Dijon et de $37 \%$ à $80 \%$ à Lille.

Tableau 2: Pourcentage d'enseignants titulaires obtenant une nouvelle académie

\begin{tabular}{lcc}
\hline \hline & $\begin{array}{c}\text { Algorithme } \\
\text { actuel }\end{array}$ & $\begin{array}{c}\text { Algorithme } \\
\text { Option 2 }\end{array}$ \\
\cline { 2 - 3 } Paris & $33,8 \%$ & $75,0 \%$ \\
Aix Marseille & $53,5 \%$ & $84,8 \%$ \\
Dijon & $39,9 \%$ & $84,8 \%$ \\
Grenoble & $45,1 \%$ & $83,7 \%$ \\
Lille & $37,2 \%$ & $80,4 \%$ \\
\hline Amiens & $31,5 \%$ & $31,9 \%$ \\
Créteil & $34,0 \%$ & $34,8 \%$ \\
Versailles & $28,7 \%$ & $30,0 \%$ \\
\hline \hline
\end{tabular}

Cette option permet donc d'améliorer significativement le mouvement global tout en garantissant que les académies d'Amiens, Créteil et Versailles, identifiées comme prioritaires, bénéficient d'un mouvement quasiinchangé. De plus, comme une partie des titulaires sortants de ces académies prioritaires sont remplacés par des enseignants à plus haut barème, l'âge moyen des enseignants affectés dans ces académies augmente : il passe de 29,6 à 30,2 ans à Créteil, et de 31,2 à 31,9 ans à Versailles. Or, comme nous l'avons déjà mentionné, plusieurs études montrent qu'une année d'expérience supplémentaire en début de carrière a un impact significatif sur la performance des élèves. Nous montrons également que d'autres indicateurs s'améliorent ou restent stables dans ces trois académies. Le nombre de néo-titulaires entrants reste parfaitement stable. Le rapport entre le nombre de titulaires sortants et entrants tend à s'améliorer, passant de 3,2 à 2,8 à Créteil par exemple (il est constant à Versailles et passe de 2,7 à 3,0 à Amiens).

Tableau 1 : Augmentation du nombre d'enseignants titulaires obtenant une nouvelle académie

\begin{tabular}{lcccc}
\hline \hline & $\begin{array}{c}\text { Algorithme } \\
\text { actuel }\end{array}$ & $\begin{array}{c}\text { EMA sans } \\
\text { académies } \\
\text { prioritaires }\end{array}$ & $\begin{array}{c}\text { EMA avec CVA } \\
\text { prioritaires } \\
\text { (option 1) }\end{array}$ & $\begin{array}{c}\text { EMA avec enseignants } \\
\text { ciblés } \\
\text { (option 2) }\end{array}$ \\
\hline Toutes disciplines & 5494 & $+40.8 \%$ & $+15.1 \%$ & $+32.3 \%$ \\
Lettres modernes & 668 & $+34.3 \%$ & $+12.9 \%$ & $+25.0 \%$ \\
Mathématiques & 645 & $+36.4 \%$ & $+2.3 \%$ & $+24.0 \%$ \\
Anglais & 512 & $+51.6 \%$ & $+19.1 \%$ & $+40.4 \%$ \\
\hline \hline
\end{tabular}

Note : EMA : échanges mutuellement améliorants. CVA : académies de Créteil, Versailles et Amiens. 


\section{La question du respect du barème}

Un dernier critère à prendre en compte lorsqu'on compare les affectations obtenues avec l'algorithme actuel et notre algorithme alternatif est le respect du barème de priorités des enseignants. En reprenant l'exemple de l'encadré 2, le barème d'un enseignant (Arthur par exemple) est respecté s'il n'existe pas d'autre enseignant ayant un barème inférieur à celui d'Arthur qui se trouve affecté dans une académie qu'Arthur préfère à celle dans laquelle il est affecté.

Deux situations existent dans lesquelles le barème d'un enseignant peut ne pas être respecté.

Premièrement, un enseignant à fort barème peut ne pas entrer dans une académie, alors que d'autres enseignants à barème plus faible restent dans cette académie car ils n'ont obtenu aucun de leurs vœux de mobilité. Dans l'exemple que nous présentons dans l'encadré 2 , le fait qu'aucun enseignant ne bouge avec l'algorithme actuel implique qu'Arthur - l'enseignant au plus haut barème - se voit refuser une affectation à Versailles ou à Bordeaux alors que Victoria et Bertrand restent dans leur académie, malgré un barème inférieur à celui d'Arthur. Cette «violation » des priorités est imputable à la garantie apportée aux enseignants de pouvoir rester dans leur académie d'origine s'ils n'obtiennent aucun de leurs vœux. L'algorithme alternatif que nous proposons, en accroissant significativement le nombre d'enseignants obtenant une nouvelle affectation, réduit d'autant cette première source de non-respect du barème. Le nombre d'enseignants titulaires dont le barème n'est pas respecté dans au moins une académie du fait d'un enseignant restant dans cette académie passe de 5094 à 1692 dans la dernière alternative que nous proposons.

La seconde source de non-respect du barème provient des échanges mutuellement améliorants. Dans notre exemple, réaliser l'échange améliorant qui existe entre Victoria et Bertrand permet d'affecter Bertrand à Versailles et Victoria à Bordeaux. Comme indiqué précédemment, la situation d'Arthur reste exactement la même avec l'algorithme actuel et dans notre alternative : Arthur reste à Amiens dans les deux cas. En revanche, pour Arthur, une nouvelle source de non-respect du barème apparaît : bien que Bertrand et Victoria disposent d'un barème inférieur à celui d'Arthur, l'échange leur permet d'obtenir une académie qu'Arthur se voit refuser. Sous la dernière alternative que nous proposons, 4702 enseignants voient leur barème non respecté du fait d'un échange. Néanmoins, il est important de garder à l'esprit que la situation de l'enseignant affecté par ce non-respect reste la même : l'algorithme actuel et notre alternative l'affectent à la même académie. En ce sens, même s'il est vrai qu'un enseignant à barème plus faible a accès à une académie qu'il désire, cet enseignant à plus faible barème ne « prend » pas la place de l'enseignant à barème plus élevé. Le non-respect de son barème n'intervient que pour satisfaire un nombre plus élevé de demandes de mutations sans que cela se fasse à son détriment. Par ailleurs, de telles situations existent déjà dans le système actuel d'affectation des enseignants, bien qu'elles restent relativement rares. Le traitement des affectations simultanées des couples, la pratique des « permutations sous la barre » lors du mouvement intra-académique et le recours aux «permutations » dans le premier degré sont des exemples dans lesquels le barème d'un enseignant peut ne pas être respecté du fait de l'entrée d'un autre enseignant à plus faible barème dans une académie.

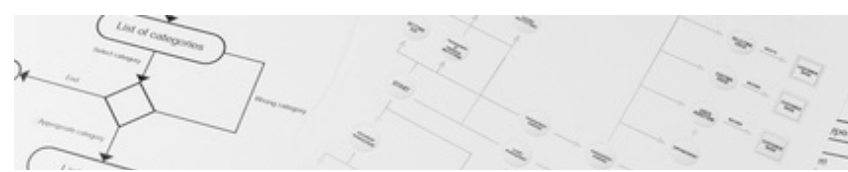

\section{Conclusion}

Nous suggérons dans cette note un changement dans la procédure d'affectation des enseignants qui permet d'augmenter considérablement le mouvement par rapport au système actuel d'affectation, sans pénaliser les académies les plus défavorisées socialement. Il est important de souligner que les résultats que nous présentons reflètent un certain nombre de choix effectués dans le cadre de nos simulations, en particulier le fait de ne considérer comme prioritaires que trois académies (Amiens, Créteil et Versailles). D'autres choix sont bien entendu possibles et notre calibrage sur les enseignants ciblés permet un large éventail de possibilités quant au degré de priorité attribué aux différentes académies. En fonction des choix politiques, la flexibilité de notre algorithme permet des arbitrages fins. Nous fournissons ainsi un outil de pilotage qui permet de faire des simulations et de tester différentes stratégies de gestion des ressources humaines propres à chaque académie.

De façon plus générale, l'objectif de cette note est de mettre en évidence l'importance de la procédure informatisée d'affectation des enseignants pour améliorer le système éducatif dans deux dimensions essentielles. D'abord, en permettant d'augmenter les perspectives de mobilité géographique des enseignants et donc, dans une certaine mesure, d'améliorer l'attractivité de la profession. Mais aussi en permettant de relever le niveau moyen d'expérience des enseignants affectés dans les académies les plus défavorisées, de manière à contribuer à la réduction des inégalités de réussite entre élèves. Nous montrons que, dans ces deux dimensions essentielles, la situation actuelle pourrait être grandement améliorée et mettons en évidence tout un spectre de possibilités dont le choix relève in fine d'une décision collective entre les différents acteurs que sont le ministère, les représentants syndicaux et les académies.

\section{Référence de l'étude}

Cette note s'appuie sur l'article : « Un nouvel algorithme pour améliorer la mobilité des enseignants sans pénaliser les académies les moins attractives », Julien Combe, Olivier Tercieux et Camille Terrier, soumis pour publication dans Éducation \& Formations.

\section{Auteurs}

- Julien Combe est doctorant à l'École d'économie de Paris.

- Olivier Tercieux est directeur de recherche au CNRS et chaire associée à l'École d'économie de Paris.

- Camille Terrier est doctorante à l'École d'économie de Paris.

\section{Soutien}

Ce travail a bénéficié d'une aide de l'État gérée par l'Agence nationale de la Recherche au titre du programme Investissements d'Avenir portant la référence ANR-10-EQPX-17 (Centre d'accès sécurisé aux données - CASD) 